\title{
The CABRI fast neutron Hodoscope: Renovation, qualification program and first results following the experimental reactor restart
}

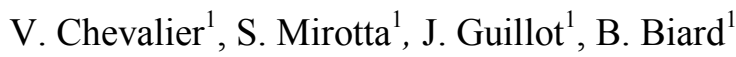 \\ ${ }^{I}$ IRSN, PSN-RES/SEREX/L2EP, Cadarache, F-13108 Saint-Paul-Lez-Durance, France
}

\begin{abstract}
The CABRI experimental pulse reactor, located at the Cadarache nuclear research center, southern France, is devoted to the study of Reactivity Initiated Accidents (RIA). For the purpose of the CABRI International Program (CIP), managed and funded by IRSN, in the framework of an OECD/NEA agreement, a huge renovation of the facility has been conducted since 2003. The Cabri Water Loop was then installed to ensure prototypical Pressurized Water Reactor (PWR) conditions for testing irradiated fuel rods.

The hodoscope installed in the CABRI reactor is a unique online fuel motion monitoring system, operated by IRSN and dedicated to the measurement of the fast neutrons emitted by the tested rod during the power pulse. It is one of the distinctive features of the CABRI reactor facility, which is operated by CEA. The system is able to determine the fuel motion, if any, with a time resolution of $1 \mathrm{~ms}$ and a spatial resolution of $3 \mathrm{~mm}$. The hodoscope equipment has been upgraded as well during the CABRI facility renovation. This paper presents the main outcomes achieved with the hodoscope since October 2015, date of the first criticality of the CABRI reactor in its new Cabri Water Loop configuration. Results obtained during reactor commissioning phase functioning, either in steady-state mode (at low and high power, up to $23 \mathrm{MW}$ ) or in transient mode (startup, possibly beyond $20 \mathrm{GW}$ ), are discussed.
\end{abstract}

Index Terms - CABRI, Hodoscope, pulse mode, RIA, fast neutron detection system.

\section{NOMENClature}

$\phi_{\text {core }} \quad$ Neutronic flux in the center of the core

$\sigma_{f, i} \quad$ Microscopic fission $\mathrm{x}$-section for the isotope i

$N_{i, \text { core }} \quad$ Atomic density of fissile material of the core

$Q_{f, i} \quad$ Q-value of the fission for the isotope i

$N_{i, \text { testrod }}$ Atomic density of fissile material of the test rod

$n_{m} \quad$ Measured counting rate

$n_{t} \quad$ True counting rate

$n_{\text {rod }} \quad$ Measured counting rate due to test rod neutrons

$n_{s c} \quad$ Measured counting rate due to scattered neutrons

$n_{b g} \quad$ Measured counting rate due to CABRI core neutrons

$\tau \quad$ Dead time of detection chain

$\vartheta \quad$ Dead time type, $0 \leq \vartheta \leq 1$

\section{INTRODUCTION}

For enhancing safety of nuclear power plants (NPP), the F Institut de Radioprotection et de Sûreté Nucléaire (IRSN) carries out some experimental programs in order to improve the understanding of the fuel behavior under severe accident conditions. One of them is the CABRI International Program, managed and funded by IRSN under an OECD/NEA agreement, launched to study Reactivity Initiated Accidents (RIA) in representative PWR conditions.

For this purpose, the renovation of the CABRI facility has been conducted by CEA, its operator. This pool type reactor is designed to submit a test rod placed in the center of the core to a RIA. The old experimental sodium loop, crossing axially the entire core, has been replaced by a pressurized water loop, so as to reproduce the thermal hydraulic conditions of a Pressurized Water Reactor (PWR), for testing the cladding of a fuel rod under prototypical conditions of RIA in PWRs.

The power transients are generated by the unique reactivity injection system of CABRI [1]. ${ }^{3} \mathrm{He}$ is previously introduced inside 96 tubes (so-called "transient rods", visible in Figure 6) located in 4 banks among the CABRI fuel rods. The very fast depressurization of this strong neutron absorber into a discharge tank, through two flow channels (low and high flow rates), is then finely adjusted to trigger the desired power pulse.

The CABRI facility is equipped with two nondestructive measurement systems operated by IRSN teams: 
- The IRIS facility, for performing X-ray radiography and tomography imaging before and after a power transient thanks to a linear electron accelerator, as well as quantitative gamma scanning analyses;

- The Hodoscope, an online fuel motion measurement system, which aims at analyzing the fuel motion deduced from the detection of fast neutrons emitted by the tested rod, in real time (with a time step of $1 \mathrm{~ms}$ ) during the transient.

In section III of this article, a brief description of the hodoscope measuring system will be given. In section 0 comparison of results performed in the old sodium loop and in the new water loop will be discussed. Furthermore, in section $\mathrm{V}$ a comparison of dosimetry measurements will be analyzed, and in section VI neutron fluxes measured by the hodoscope will be compared to those obtained by Monte-Carlo simulations. In section VII dead time analysis of the different detectors will be investigated as well. Finally, in VIII current studies will be presented, and conclusion is given in section IX.

\section{GENERAL DESCRIPTION OF THE SYSTEM}

The CABRI reactor has been equipped with the hodoscope since 1977. This system measures in real time the behavior of the test rod in the center of the CABRI core during the transient. The hodoscope is able to quantify the amount of fuel ejected in the milliseconds following failure, time-dependent axial fuel mass distributions and to follow the fuel clusters after failure. In addition, the initial state of the test rod is precisely known thanks to the hodoscope measurement of the fissile length and of the axial power profile.

The hodoscope centerpiece is a $3 \mathrm{~m}$ steel collimator (in green in Figure 1) of 3 columns and 51 rows, so 153 channels. Behind each one of these line-of-sights, a ${ }^{237} \mathrm{~Np}$ fission chamber detector $(\mathrm{FC})$ and a proton recoil proportional detector (PR) measure the fast neutrons coming from the core and test rod. Two different technologies of detectors are used in CABRI in order to follow all the experiments from low power $(\sim 50 \mathrm{~kW})$ up to $20 \mathrm{GW}$. Proton recoil counters are better suited for low power (up to $\sim 1 \mathrm{GW}$ which means 2.5 million impulsions per second), thanks to a higher efficiency and no discrimination of the $\mathrm{y}$ noise, and ${ }^{237} \mathrm{~Np}$ fission chambers are used for higher power. The Proton Recoil detectors are operated at 2500 Volts and an energy threshold at $400 \mathrm{keV}$. The ${ }^{237} \mathrm{~Np}$ fission chamber detector (FC), operated at $600 \mathrm{~V}$, have low gamma sensitivity and an energy threshold of $650 \mathrm{keV}$. The signal acquisition rate may be adjusted between $1 \mathrm{~ms}$ and $10 \mathrm{~s}$ according to the count rate. The hodoscope collimator has three different degrees of freedom, for adjusting its position in front of the test rod. The distance from the collimator nose to the core axis may be adjusted manually in translation, while two Direct Current Motors are used to move the collimator in verticality and rotation. Each one of the 306 detectors is linked by a $\mu$-metal cable to its electronic chain placed $30 \mathrm{~m}$ far from the detector. These electronics have been renewed taking benefit from the renovation period [2].

In order to keep the noise/signal ratio as low as possible, the CABRI driver core is traversed radially by the irradiation channel. In that way, none of the fuel rods of the core are in direct view of the hodoscope detectors. Nevertheless, the noise to signal ratio remains low and requires specific data treatment algorithms.

The collimator was installed in order to pixelize the test rod placed in the center of the reactor. Each detector looks at a slice of the test rod. In steady state conditions, the signals measured by all detectors are stable, whereas in transient conditions (i.e. a RIA pulse) they may vary according to a fuel displacement, a fuel densification (yielding to an increasing signal), or a fuel dilution or ejection (yielding to a decreasing signal).

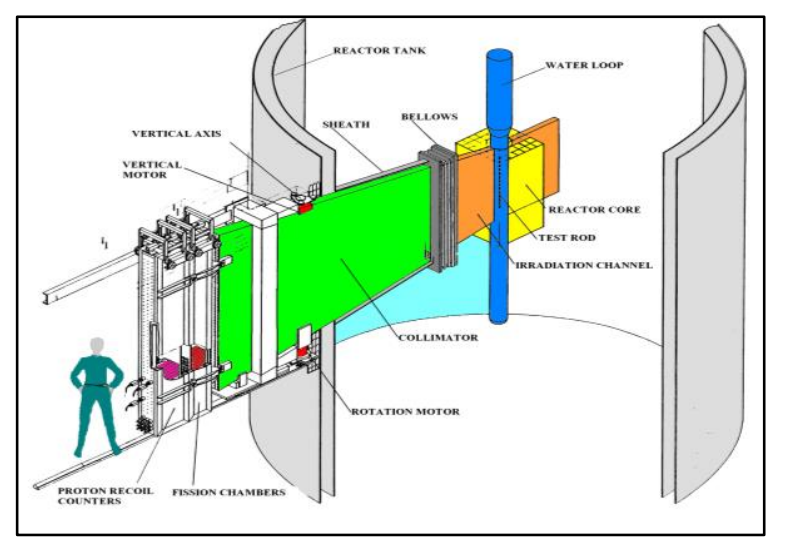

Figure 1: Hodoscope detector system

\section{COMPARISON OF NEW WATER LOOP WITH SODIUM LOOP}

The CABRI restart campaign, started in October 2015, could be split into four main kinds of experiments:

1. Low power experiments - part 1, performed to measure neutronic parameters of the reactor. In this part the hodoscope was not used because the sensitivities of PR and FC are too low to measure signals when core power is limited to $10 \mathrm{~kW}$; 2. Low power experiments - part 2, in this second part reactor power has reached $80 \mathrm{~kW}$; at this power level the hodoscope has given first results;

3. High power experiments in steady state conditions, these experiments has taken the core power up to $23 \mathrm{MW}$, in order to calibrate the core detectors [3];

4. High power experiments where perfomed to reproduce power pulses up to $21 \mathrm{GW}$.

Results obtained during the CABRI restart campaign (phase 3 and 4) are compared to those obtained in the past, in order to observe potential modification in detector response. This comparison allows to determine whether the hodoscope counting chain was damaged or not during the facility renovation. Indeed, some major works were conducted during the renovation of the facility which led to a 12 year shut off period for the hodoscope, including seismic reinforcement in the immediate proximity of the detectors and the collimator (kept in place during the renovation) and of the electronic chains which had to be moved away and back.

In this campaign the device positioned in the center of the CABRI core inside the Water Loop (WL) was equipped with a dummy lead rod which does not influence the neutronic profile in the center of the core. In order to observe the 
differences due to the new Water Loop, we have compared these results to those obtained in the last startup campaign of CIP02 (S9CIP02) experiments on 2002. S9CIP02 experiment was chosen because of its experimental conditions, which were comparable to that of a test realized in the new commission campaign. In both cases, given the absence of a real fuel rod in the test cell, the hodoscope recordings are then only background signal coming from the core fuel and not "useful" neutrons from a test rod.

In these experiments, the same depressurization parameters, ${ }^{3} \mathrm{He}$ initial pressure (hence the same control rod position) and initial power were set.

Next sub section will focus on the axial power profiles measured by the hodoscope in steady state conditions (during a $100 \mathrm{~kW}$ power plateau) with both FC and PR detectors and in transient conditions (during power peak transient) just for FC.

\section{A. Power profiles at $100 \mathrm{~kW}$ steady state conditions}

Power profiles may be compared in steady state conditions with ${ }^{3} \mathrm{He}$ in the transient rod, before triggering the pulse.

Uncertainties in this case are much higher because of the low counting rate at $100 \mathrm{~kW}$. Such acquisitions are performed at this low power level for determining the initial and final state of the test rod without heating it too much. The acquisition rate is then tuned to its minimum value $(1 \mathrm{~ms})$ to follow the power transient.

An average over three columns (for FC in the same row) has been calculated, taking into account neither a dead time correction (at this low counting rate the detectors are in their linear range) nor a sensitivity coefficient (would be required only for a quantitative analysis).

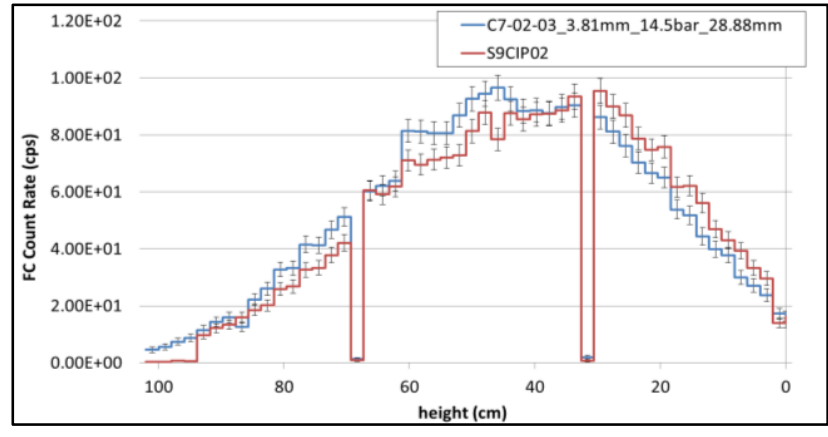

Figure 2: FC axial power profiles in Steady State Condition

The comparison is presented in Figure 2 for $\mathrm{FC}$ and in Figure 3 for PR. Axial height is counted from the bottom of the CABRI core fuel rods. Two detector chains have a low counting rate because two line-of-sights are filled with stainless steel in order to strengthen the collimator. The FC comparison is consistent, with differences inside a $2 \sigma$ confidence interval. No degradation of the measurement system can be observed. This result makes feel confident about the global response of the system in steady state condition at low power.

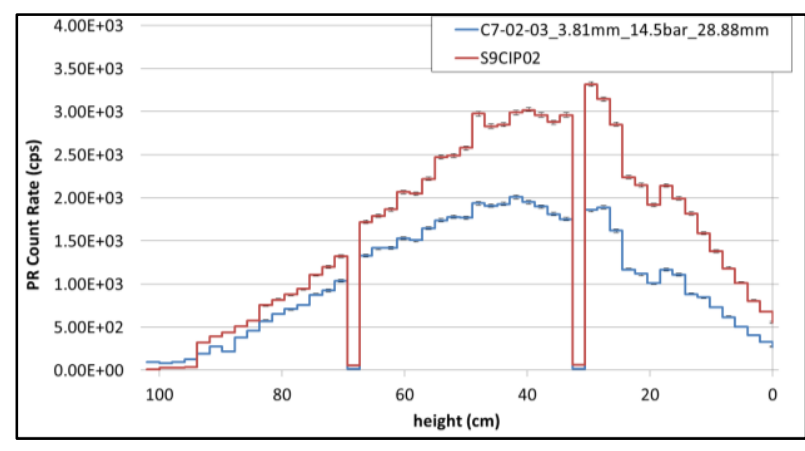

Figure 3: PR axial profiles in Steady State Condition

For PR detectors, a decreased measured signal is observed. This is due to the refilling of the PR detectors with $\mathrm{CH}_{4}$ (all PR detectors are interconnected), increasing the gas circuit pressure to 1.23 bar instead of the past 1.05 bar. Decreasing the measured counting rate allows to reach a higher power before saturation of PR detectors.

\section{B. Power Profile in transient conditions}

It is now possible to compare axial power profiles during power transient where the count rate is higher, hence the uncertainties are lower. Profiles presented in Figure 4 are calculated just at the maximum of the power transient, and show a normalized axial power profile obtained in the last experimental campaign compared again to S9CIP02.

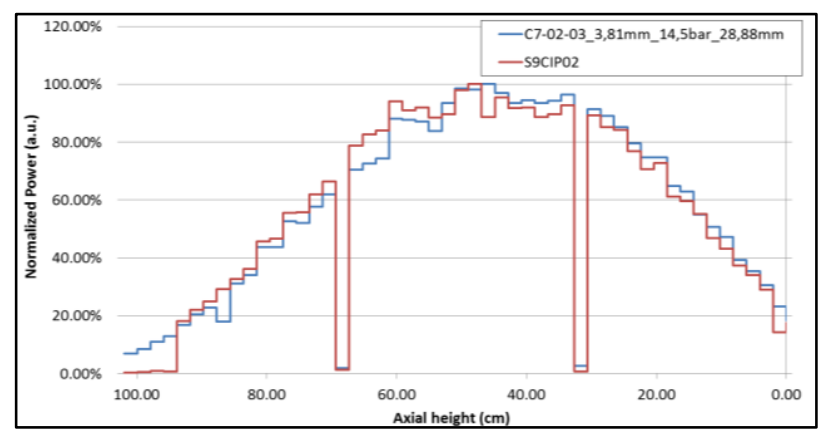

Figure 4: Normalized FC axial power profiles in Transient Condition (Max Power of 5 GW)

Uncertainties are less than $0.5 \%$ and are not appreciable in the figure.

As for the previous comparison, results shown in Figure 4 are neither evaluated with a dead time correction nor a sensitivity correction, which are not necessary for this qualitative comparison.

At this step, the general functioning of the hodoscope system is demonstrated. Indeed, during the power steady states and the pulses, it was observed:

- The correct operation of all measuring channels (detectors, associated electronics) whatever the power level and the acquisition rate,

- The correct operation of the remote acquisition module, 
- The proper functioning of real-time tracking of all channels on the new acquisition station and monitoring $\mathrm{PC}$,

- The correct operation of the software and numerical tools dedicated to preparation, acquisition and analysis.

\section{COMPARISON WITH DOSIMETRY MEASUREMENT}

The CABRI commissioning campaign included several experiences with dosimetry measurements [4]. Wire dosimeters were placed at several known elevations in the center of the test cell to measure the axial power profile of the CABRI core. These results were compared to those obtained with the hodoscope PR detectors in order to determine their qualitative response. On Figure 5 are shown the axial profile measured with the PR detectors of the hodoscope and the dosimeter counting rates. The power plateau for this experience was set at $30 \mathrm{~kW}$ and the measurements are consistent.

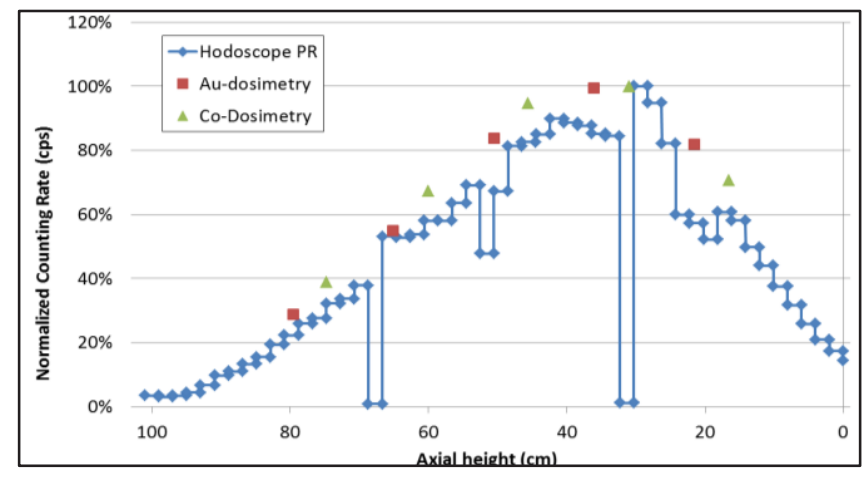

Figure 5: Hodoscope power profile vs. dosimetry in Steady State condition

An important aspect to observe in this graph is that hodoscope PR detectors measure fast neutron flux coming from the core, whereas dosimeters measure thermal and epithermal fluxes of the core. Consistency between these curves is due to a constant ratio between thermal and fast flux during steady state conditions. This assumption is also verified in transient conditions.

\section{COMPARISON WITH NUMERICAL SIMULATION}

In order to solve the Boltzmann Equation, all deterministic code needs to do some approximation. For CABRI reactor, deterministic codes do not give very satisfying answers. Therefore, Monte Carlo code have been used to determine

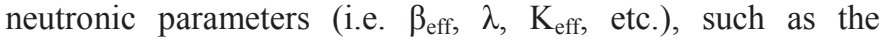
MORET code developed by IRSN [5]. The MORET model of CABRI core is shown on Figure 6.

In this section, results obtained with MORET 5.B.1, using JEFF3.1 libraries, are compared to experimental results. Monte Carlo codes give results in steady state conditions, so a comparison has been carried out with experimental results obtained during a $23 \mathrm{MW}$ power plateau.

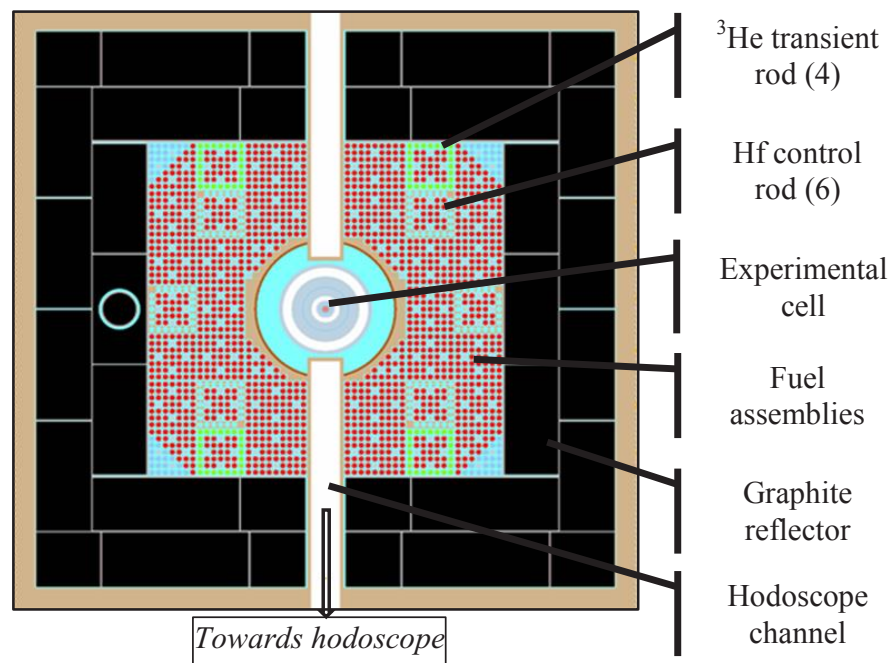

Figure 6: MORET model of the CABRI core

For this comparison, dead time correction (as detailed in section VII) and sensitivity coefficient estimated in 1994 were used in order to obtain physical results.

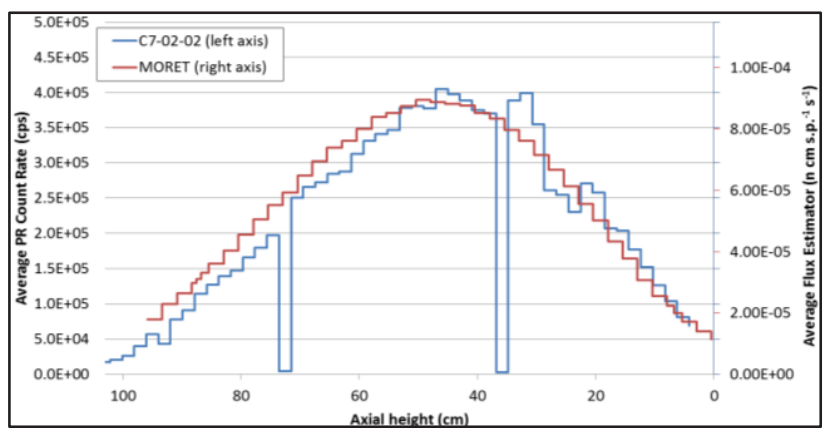

Figure 7: MORET 5 compared to PR profile in Steady State Condition

As we can see in Figure 7, Monte Carlo predictions (right axis) are consistent with experimental measurements (left axis). This result makes feel confident with calculated results, and especially the coupling factor calculation expressed in Eq.(1).

$C F=\frac{P_{\text {core }}}{P_{\text {testrod }}}=\frac{\sum_{i} \phi_{\text {core }} \cdot \sigma_{f, i} \cdot N_{i, \text { core }} \cdot Q_{f, i}}{\sum_{i} \phi_{\text {core }} \cdot \sigma_{f, i} \cdot N_{i, \text { testrod }} \cdot Q_{f, i}}$

This coupling factor estimation is of crucial importance for the experiment, since it allows the estimation of the energy that will be deposited into the test rod during a power transient, based on the core power measurement.

\section{DEAD TIME CHARACTERIZATION}

The analysis of the first commissioning tests also allows the characterization of the dead time of detectors. The PRs as well as the FCs are all operated in pulse mode. This is possible because of the important distance between the core and the 
detectors. As shown in Figure 8, for the FC, when the counting rate arises up to 4 million impulses per second, the detector's response becomes nonlinear. In order to solve this problem, a dead time correction must be applied.

This correction is applied just to FC detectors because PR detectors become useless at high power.

Till today, for dead time correction of FC detectors Eq. (2) was used regardless the counting rate. This correction is in great accord with the true counting rate when dead time losses are smaller than $\sim 15 \%$. Beyond this limit this correction is not efficient. This is because Eq. (2) describes the behavior of an electronic chain with a non-parallelizable dead time. [5]

$$
n_{m}=\frac{n_{t h}}{1+\tau \cdot n_{t h}}
$$

In reality both $\mathrm{FC}$ and $\mathrm{PR}$ detectors present an intermediate behavior between paralyzable and nonparalyzable dead time, which is characterized by a parameter $\vartheta$. Therefore, the more complex Eq. (3) has to be used for a good evaluation of dead time losses.

$$
n_{m}=\frac{n_{t} \cdot \vartheta}{e^{n_{t} \cdot \tau \cdot \vartheta}+\vartheta-1}
$$

Using this new approach, $\vartheta$ and $\tau$ have to be evaluated. In order to do that, the following methodology was used:

1) For low counting rate (i.e. $10 \%$ of losses) Eq.(2) gives us dead time of the electronic chain ${ }^{1}$;

2) Then Eq.(3) is solved to determine theta using experimental results at high counting rate. Indeed, the true counting rate according to the measured one has to be known;

3) These two parameters $(\tau, \vartheta)$ are used in Eq.(4) that is a Taylor expansion of rearranged Eq.(3) in order to find the true counting rate. Indeed, the true counting rate according to the measured one has to be known.

$$
\begin{aligned}
n_{t}=n_{m} \cdot\left[1+n_{m} \cdot \tau\right. & +\left(1+\frac{\vartheta}{2}\right) \cdot\left(n_{m} \cdot \tau\right)^{2} \\
& \left.+\left(1+\frac{3}{2} \vartheta+\frac{\vartheta^{2}}{6}\right) \cdot\left(n_{m} \cdot \tau\right)^{3}+\cdots\right]
\end{aligned}
$$

With this methodology all 306 dead time parameters were obtained.

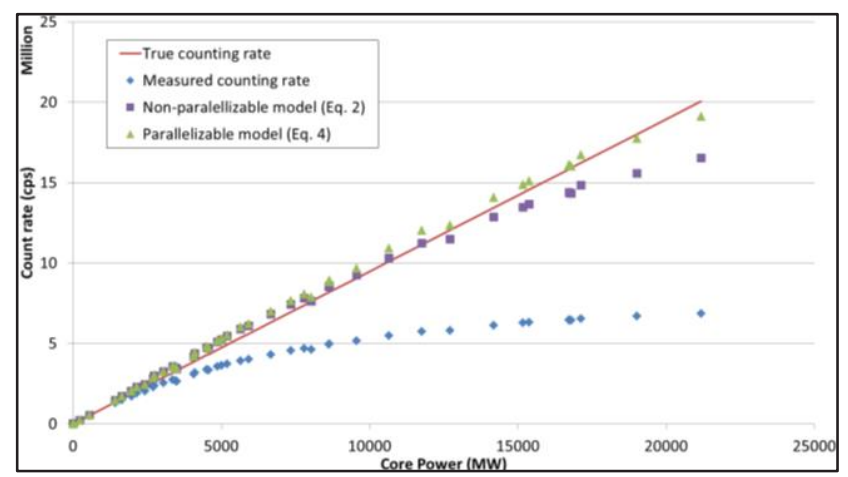

Figure 8: Comparison of dead time correction algorithms

\footnotetext{
${ }^{1}$ These values are consistent with those evaluated by the double oscillators methodology [6]
}

As we can see in Figure 8 the counting rate reevaluated after dead time correction is consistent with the true counting rate. Results found for three FCs are summed up in Table 1 for three detection chains. This analysis must be realized for each detector of the hodoscope system. This dead time correction allows the hodoscope to monitor with a good linearity even the most powerful transients of CABRI.

Table 1- DEAD TIME CHARACTERISTIC FOR THREE FC

\begin{tabular}{cccc}
\hline \hline & $\begin{array}{c}\text { Upper row } \\
(\mathrm{FC} 189)\end{array}$ & $\begin{array}{c}\text { Middle row } \\
(\mathrm{FC} 237)\end{array}$ & $\begin{array}{c}\text { Bottom row } \\
(\mathrm{FC} 278)\end{array}$ \\
\hline$\tau$ & $82.6 \mathrm{~ns}$ & $85.4 \mathrm{~ns}$ & $87 \mathrm{~ns}$ \\
\hline$\vartheta$ & 0 & 0.134 & 0.572
\end{tabular}

\section{PERSPECTIVES}

In June 2017 a calibration campaign is planned in order to adjust the sensitivity coefficients for both FC and PR detectors. This campaign will be split in two parts:

- The Collimator Alignment campaign will aim at verifying the collimator position viewing the rod in the test cell;

- The Detector Calibration campaign will be devoted to the determination of the sensitivity coefficient for each FC and PR detector.

This campaign is necessary to obtain absolute quantitative measurements with the hodoscope system.

\section{A. Aligning the hodoscope collimator}

The Hodoscope Collimator may be motioned according to different degrees of freedom presented in Figure 9, so as, zaxis rotation and $y$-axis rotation allow to align the lines of sight of the hodoscope with the test rod fuel stack.

The first part of the campaign will be dedicated to the rotation alignment (c.f. z-axis rotation). In this way, it is possible to look at the test rod through the collimator. The graph presented in Figure 10 is obtained with this experiment. The second part of the campaign consists on moving the collimator under the y-axis in order to align with the vertical axis as the core. This verification will be based on the counting profiles obtained in the left, middle and right columns of the hodoscope. If the alignment is good, the fuel rod may be seen by only one column at a time. The distance between the nose of the collimator and the test rod will be verified with the measurement of the fissile length of the fuel stack of a fresh fuel rod placed into the experimental cell. Since the lines of sight are not parallel but convergent, this measured length will vary according to the distance of the collimator from the fuel rod.

This campaign will follow an iterative way: a power plateau of $\sim 10 \mathrm{MW}$ will be scrutated by the hodoscope while rotating the collimator, in front of the test rod. Once the data analyzed, a correction will be determined for the vertical motor position. A new power plateau will be performed to check this new position, etc.

When the global alignment is alright, it will be possible to determine the optimal measuring position. Indeed, by rotating 
the collimator, the operators may choose which column does see the rod and which one does not.

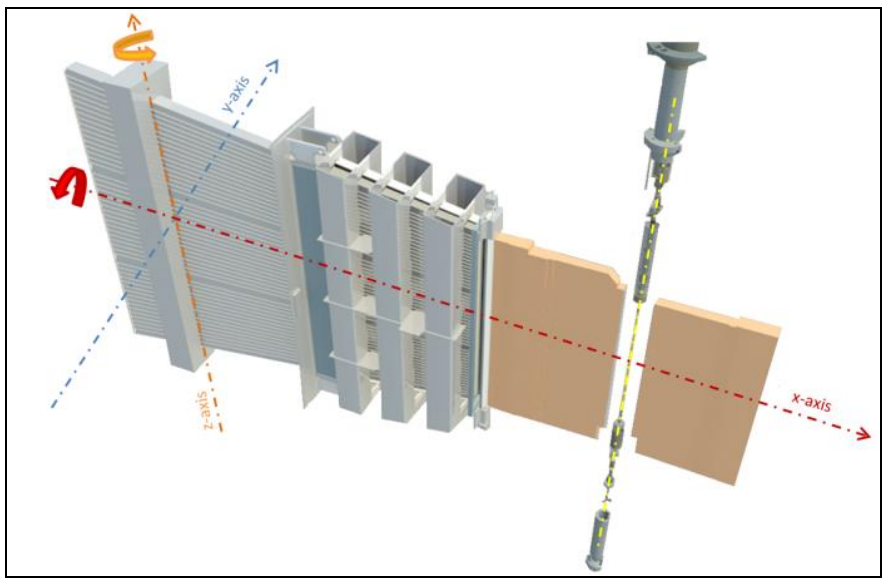

Figure 9: Hodoscope collimator movements

When a detector is in front of the test rod, its counting rate will be given by:

$$
n_{m}=n_{\text {rod }}+n_{s c}+n_{b g}
$$

When the collimator is shifted away from the test rod axis, hodoscope detectors will count just background and scattered neutrons coming from the CABRI core, as shown by Eq.(6).

$$
n_{m}=n_{s c}+n_{b g}
$$

Figure 10 shows the response of one row when the collimator is rotated in front of the test rod. When the collimator is in front of the test rod a maximum will be obtained. When it is far from the test rod just background and scattered neutrons are measured.

The standard position of the hodoscope, named "split" position, consists in placing the collimator between left and central row. By this way, with left and central row we are able to measure counting rate expressed in Eq.(5), whereas with the right column it is possible to measure neutrons coming from the core only (c.f.Eq.(6)).

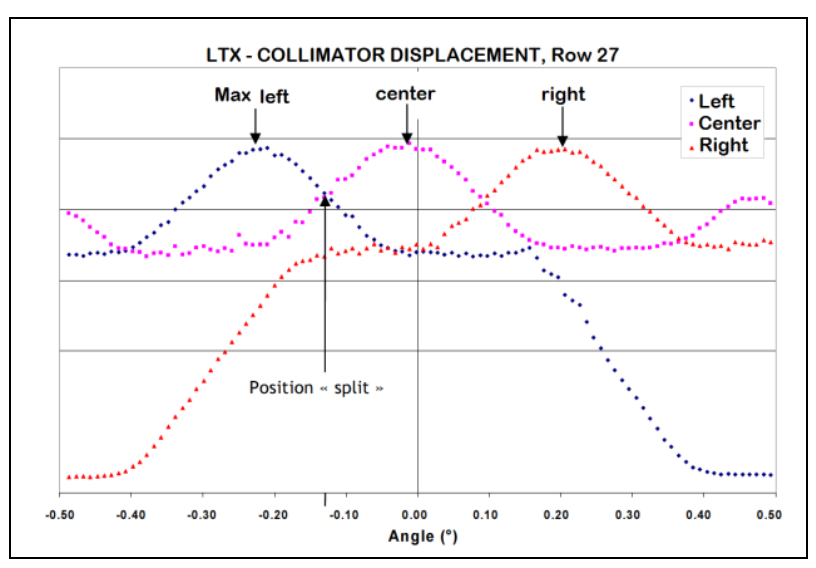

Figure 10: Measured counting rate for left, center and right detectors of a common row during collimator rotation
The split position is preferred to a more classical position when just one column looks at the test rod because in this way signal variations are seen by two columns simultaneously reducing uncertainties.

This campaign should be performed each time a test device will be loaded in the experimental cell with a new test rod.

\section{B. Detectors Calibration Campaign}

After the collimator alignment, the calibration campaign will be performed to adjust the detectors efficiency. In order to evaluate these efficiencies (different for each detector), the experiment consists first in irradiating a fresh $\mathrm{UO}_{2}$ fuel rod in CABRI for a couple of hours (duration limited by the core cooling system capacities) at a constant power while monitoring fast neutrons emitted from this fuel rod by the hodoscope system. After the irradiation, the axial profile of the non-volatile fission products created during the irradiation of the test rod will be measured on the IRIS facility by $\gamma$ spectroscopy analysis.

Although the direct measurement with the hodoscope is based on fast neutrons while the subsequent IRIS counting is based on gamma measurement, this comparison is relevant assuming both particles are proportionally generated by the thermal fissions of $\mathrm{UO}_{2}$. Each fission produces indeed $\bar{v}$ neutrons, which are directly detected by the hodoscope, some prompt $\gamma$ and fission products that will be detected in IRIS. $\gamma$ emitted by the radioactive decay of fission products will give us the amount and the location of the fission products. In this way it is possible to build the axial profile of CABRI core. Efficiency coefficients will finally be obtained by comparison of this profile with those measured with the hodoscope system.

\section{CONCLUSION}

After a long standby and renovation period, the restart campaign of the CABRI reactor has allowed to verify the good operability of the hodoscope detectors, their electronic chains and the command and control system, and verifying its operating domain. It was also useful to obtain the detector response at different core power levels, up to $23 \mathrm{MW}$ in steady-state mode and up to more than $20 \mathrm{GW}$ in transient mode. Furthermore, with these experiments the dead time parameters of each detector were characterized. The hodoscope is now ready to be aligned and calibrated thanks to a dedicated campaign that will be carried out in order to adjust the sensitivity coefficients for both FC and PR detectors.

\section{REFERENCES}

[1] B. Duc, B. Biard, P. Debias, L. Pantera, J.-P. Hudelot and F. Rodiac, "Renovation, improvement and experimental validation of the Helium-3 transient rods system for the reactivity injection in the CABRI reactor," IGORR 2014 Conference, Bariloche, Argentina, November 2014.

[2] S.Mirotta, J.Guillot, V.Chevalier and B.Biard, "Qualification and characterization of electronics of the fast neutrons Hodoscope detectors using neutrons from CABRI core," In ANIMMA2017 Conference Proceedings, 
Liege, June 2017.

[3] J. Lecerf, Y. Garnier, J. Girard, C. Domergue, L. Gaubert and C. Manenc, "Study of the linearity of CABRI experimental ionization chambers during RIA transients," in ANIMMA 2017 Conference Proceedings, Liege, June 2017.

[4] J. Lecerf, Y. Garnier, J. Hudelot, J. Girard, C. Domergue, L. Gaubert, M. Maillot, C. Manenc and S. Mirotta, "Analysis of the dosimetry campaign performed during the CABRI commissioning tests," In ANIMMA2017

Conference Proceedings, Liege, June 2017.

[5] G. F. Knoll, "Radiation Detection and Measurement," John Wiley and Sons, 3rd edition, 1999.

[6] J. Gostely et E. Carnal, «La methode des deux oscillateurs pour la mesure du temps mort dans l'instrumentation nucléaire,» Nuclear Instruments and Methods, Vol 150, Iss 3, Pgs 459-464, 15 April 1978.

[7] B. Cochet, A. Jinaphanh, L. Heulers and O. Jacquet, "Capabilities overview of the MORET5 Monte Carlo code," Annal of Nuclear Energy, Vol 82, Pgs 74-84, August 2015. 